\title{
Model Based Data Transmission: Analysis of Link Budget Requirement Reduction
}

\author{
Jeremy Straub \\ Department of Computer Science, University of North Dakota, Grand Forks, USA \\ Email: jeremy.straub@my.und.edu
}

Received July 20, 2012; revised August 22, 2012; accepted September 23, 2012

\begin{abstract}
Communications capability can be a significant constraint on the utility of a spacecraft. While conventionally enhanced through the use of a larger transmitting or receiving antenna or through augmenting transmission power, communications capability can also be enhanced via incorporating more data in every unit of transmission. Model Based Transmission Reduction (MBTR) increases the mission utility of spacecraft via sending higher-level messages which rely on preshared (or, in some cases, co-transmitted) data. Because of this a priori knowledge, the amount of information contained in a MBTR message significantly exceeds the amount the amount of information in a conventional message. MBTR has multiple levels of operation; the lowest, Model Based Data Transmission (MBDT), utilizes a pre-shared lower-resolution data frame, which is augmented in areas of significant discrepancy with data from the higher-resolution source. MBDT is examined, in detail, herein and several approaches to minimizing the required bandwidth for conveying data required to conform to a minimum level of accuracy are considered. Also considered are ways of minimizing transmission requirements when both a model and change data required to attain a desired minimum discrepancy threshold must be transmitted. These possible solutions are compared to alternate transmission techniques including several forms of image compression.
\end{abstract}

Keywords: Spacecraft Communications; Data Compression; Satellite Communications; Link Budget Reduction; Image Format

\section{Introduction}

Any space mission must contend with degradation of communications performance as the spacecraft moves further from Earth. Commonly, larger transmitting or receiving antennas or increased transmission power are utilized to maintain the required data rate. However, for small satellites (such as SmallSat and CubeSat-class craft) power, mass and volume constraints preclude significantly boosting the signal strength, and thus effective data rate, through transmission antenna or power augmentation. The budgets of many of these programs, similarly, preclude procuring or leasing access to the larger antennas that would be required to increase signal strength/ reception and, thus, data rate from the ground station side.

For many missions, the science utility of the mission can be conceptualized as being a function of the data that is received by the ground station. The amount of data that is received, can be constrained by the sensors and equipment that generate it. However, in many cases these sensors can generate far more data than can be processed and transmitted by the spacecraft. Thus, onboard processing capabilities and transmission capabilities become the bottleneck. Model-Based Data Transmission (MBDT) is not a panacea for this problem, as it trades the increased consumption of onboard processing resources for a decrease in the amount of bytes of data transmission required to transmit a given amount of information.

Model-Based Data Transmission is the lowest level of Model-Based Transmission Reduction (MBTR), which is a set of methods for transmitting higher-level and more meaningful messages. Each MBTR message conveys significantly more data than a message under the conventional transmit-each-data-element approach. Each level of MBTR requires progressively more onboard computational resources to perform.

\section{Background}

Bozzi et al. [1] state that data compression is not generally used on deep-space missions. Despite this, smaller spacecraft, such as SmallSats and CubeSats, will generally require some form of compression, particularly when they go beyond Earth orbit. This is necessitated by the small spacecraft size and the level of data transmission required to achieve mission objectives.

Compression has been implemented on previous mis- 
sions. The Helioseismic Magnetic Imager (HMI) Instrument aboard the Solar Dynamics Observatory, for example, has utilized compression as part of its mission design [2]. The HMI uses hardware image processing boards to crop images and perform lossless compression. The design for the High Time Resolution Spectrometer (HTRS) instrument on the International X-ray Observatory, utilizes compression for lists of time stamp, silicon drift detector and energy data [3]. Planning simulations indicated that up to a $50 \%$ compression level was achievable for this type of data, when CPU-intensive compression software was utilized.

In response to a failure of the high-gain antenna, the Galileo mission utilized an image compression approach similar to that used by the JPEG standard [4]. An order of magnitude of improvement in the level of images and other data was achieved, compared to pre-failure estimates of the transmission capabilities of the low-gain antenna [5].

Faria et al. [6] compared, for satellite imagery application purposes, a variety of image compression techniques. Their work proffers that compression ratios of up to 353.5 may be possible; however, the quality (and thus suitability) of imagery at these high levels of compression is unclear.

\section{MBTR Framework}

The MBTR paradigm (presented in [7,8]) is a "game changing" approach to space communications. Unlike traditional communications models which transmit numerous discrete or related data elements for analysis on Earth, MBTR performs onboard analysis. MBTR consists of four different levels of onboard processing activities. Each progressively higher level encapsulates the activities of the lower levels, requiring more onboard processing capabilities and producing messages which convey a greater level of meaning per unit of data transfer.

The lowest level of MBTR is Model-Based Data Transmission (MBDT), which is the primary subject of this paper. Under MBDT, both the spacecraft and the ground station begin with a pre-shared low-resolution version of the data of interest. For imagery, for example, this might be an image taken by an Earth-based or Earthorbiting satellite. This low-resolution data set is utilized as a model to which changes are applied. Transmission requirements are thus reduced because only the changes (not the whole data set) must be transmitted to mission scientists.

The second level of MBTR is Model-Based Data Analysis (MBDA). Under MBDA, context-aware data analysis is performed to identify the most important areas of model discrepancy for transmission. This requires contextual understanding and a framework under which to evaluate the importance of each prospective model change (which may or may not include the discrepancylevel-based metric used in MBTR as well as other metrics).

Model-Based Result Transmission (MBRT), the third level of MBTR, extends MBDA through additional analysis. Under MBRT, data is prioritized based on the particular thesis (and the associated priority of the thesis) that it helps support or refute and the judged-level of support or refutation that it provides. MBRT, thus, begins to look at data from a mission utility maximization perspective.

The highest level of MBTR, Model-Based Findings Transmission (MBFT), maximizes the value of the communications link. Under MBFT, the base model of the phenomena of interest is revised based on data-driven changes. The model updates are reviewed to determine whether they are relevant to the mission-objective-driven findings. Differences between the a priori projected findings and those arrived at through proximal and in-situ data collection are prioritized based on their significance. Updated findings are transmitted to ground controllers along with supporting data that allows the proper operation of the onboard autonomous software to be validated. This validation data also serves to allow the scientific community to conduct a rigorous review of mission operations.

\section{Discussion of Data versus Transmission Requirements}

To effectively compare the value provided by different levels of MBTR (described in Section 3), it is necessary to create two definitions of data. The first, transmitted data, is (as the name suggests) the amount of data sent over the communications link, ignoring transmission protocol overhead. The second, effective data, is the amount of knowledge/information that is provided by the transmission. This is estimated, for comparison purposes, through ascertaining the amount of data that would be required to be transmitted with a conventional approach to provide the knowledge obtained.

For illustrative purposes, under MBFT, a finding might be transmitted requiring 10 kilobytes of finding data and 1.5 megabytes of validation data. Presuming that the spacecraft was configured to send only $1 \%$ of data for verification, under a conventional approach, this would have required 150 megabytes of data. Thus, the transmitted data would be 1.51 megabytes and the effective data would be 150 megabytes.

\section{Discussion of MBTR Advantages and Disadvantages}

MBTR, when implemented properly, can dramatically increase the science return of deep-space and even some 
Earth-orbiting missions. At its highest level of operation, it removes most of the data transmission required and simply transmits scientific findings and limited data to justify the conclusion. This is akin to a journal article: the experiment and conclusion are presented along with summary data and examples to explain the experimental process and convince the reader of its proper implementation. However, unlike a journal article, where the researcher retains the data for later review or re-use by others in the scientific community, the data supporting the MBTR conclusions survives only as long as the mission craft-and there may be insufficient time before the craft's intended or incidental end-of-life to bring back even a portion of the data on which a conclusion is based. Onboard storage re-use requirements may even necessitate the loss of this data prior to the end of the mission. This is the fundamental dilemma of the MBTR paradigm.

Findings from MBTR will likely only be a component of understanding a particular phenomenon, and will need to be combined with other observations (including, perhaps, other sets of MBTR findings) to produce a level of insight comparable to the typical journal article. None the less, the unavailability of the underlying data pits the standard scientific process against a desire for maximizing scientific understanding. One can either limit research to what the link budget can support full data transfer for-or embrace a new model where scientific rigor is based on testing and trusting the process-but not being able to review each and every piece of individual data.

Fortunately, MBDT does not suffer from this particular problem. With MBDT, all of the data is still returned. However, the level of minimum guaranteed quality (MGQ) is the subject of decision-making. Later sections present the trade-off between guaranteeing a higher level of MGQ and reducing transfer requirements. Conventional wisdom [1] seems to be that no level of loss should be accepted; however, this ignores the fact that the resolution of the image is inherently a quality standard. Just as data is lost through using lower than sub-atomic-scale resolution, data is lost through compression and other processing as well. MBDT, however, can be configured to attempt to maximize the transmission of important data through providing priority to dissimilar pixels (or pixel clusters) in an area-thus increasing resolution on anomalies while limiting the transmission cost for areas of minor fluctuation.

\section{MBDT for Image Data}

Many space missions transmit image data. This data is analyzed for scientific analysis purposes and it is also made available to members of the public for viewing. Thus, transmitted image data must, generally, be both highly accurate and pleasant to view. Techniques which diminish data accuracy while maintaining smoothness (and other viewing pleasantness metrics) or which maintain accuracy while causing pixilation (or other artifacting which makes the image appear less natural) are not, generally, acceptable. Figures 1-3 allow a comparison of the source image to images that are visually pleasing and more accurate, but less visually pleasing.

\subsection{Overview}

Two uses for MBDT for image data are examined. In the

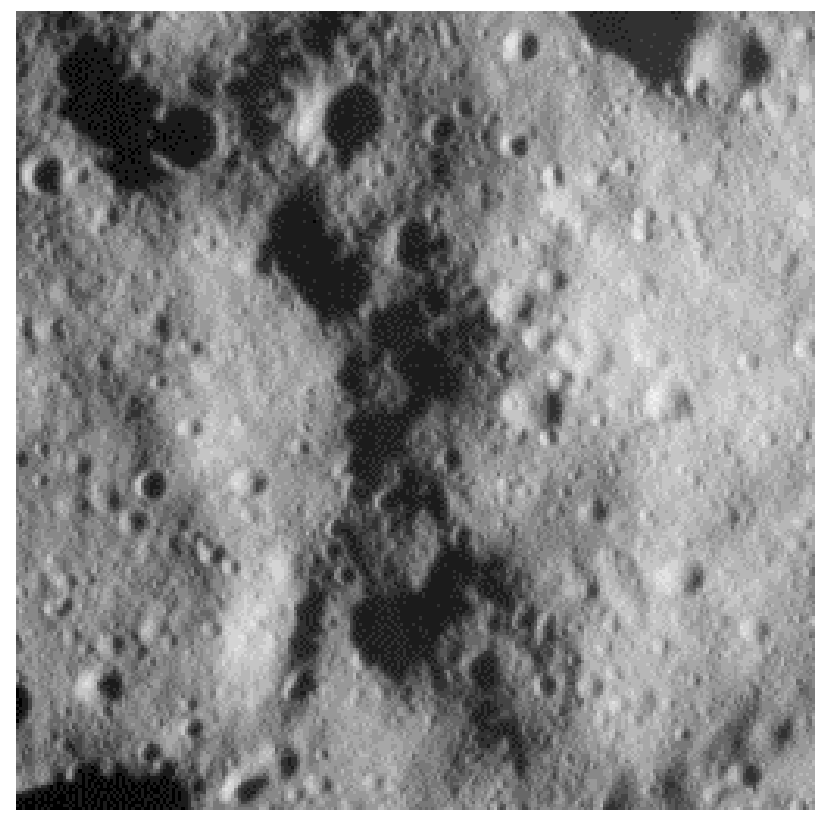

Figure 1. Source image.

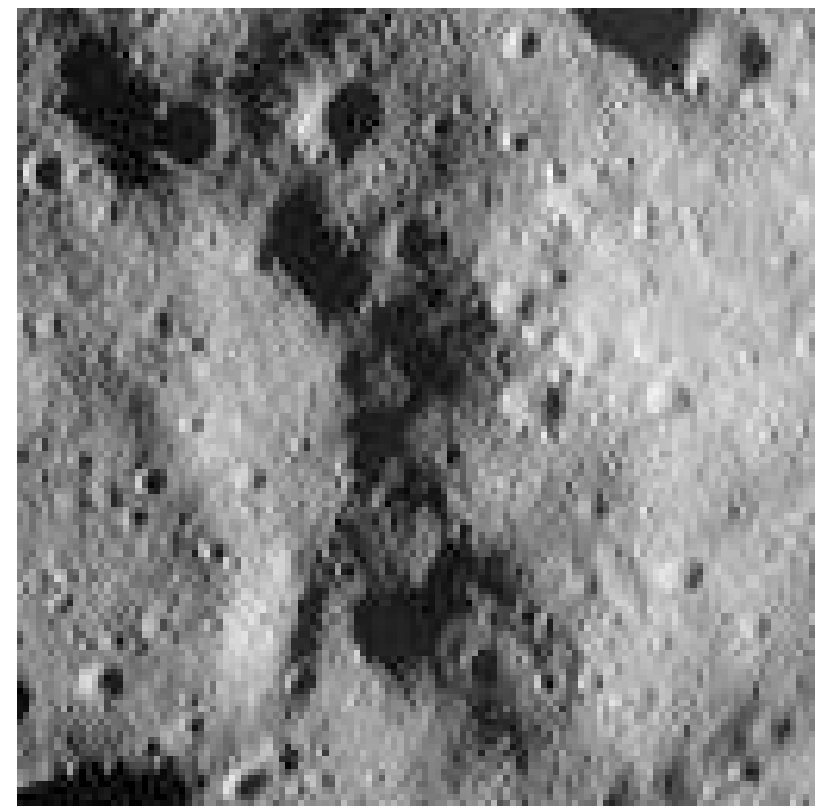

Figure 2. JPEG image, up-scaled from $50 \% \times 50 \%$ of original height and width, 70 level of storage quality. 


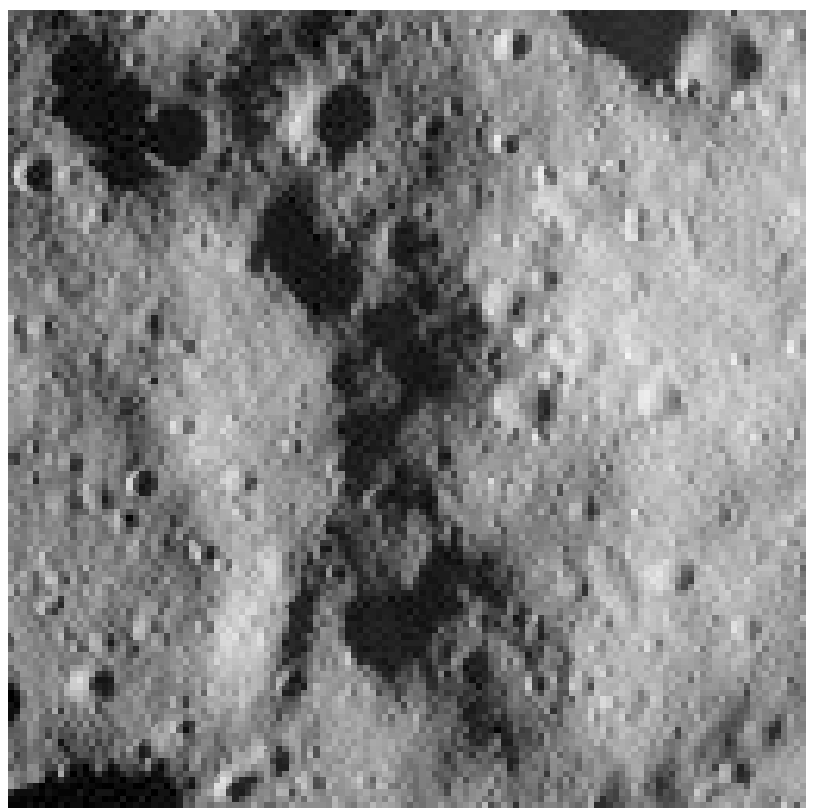

Figure 3. JPEG image, storage quality 90, enhanced from $50 \% \times 50 \%$ of original height and width to $20 \%$ MGQ.

first, a shared a priori low-resolution image exists which is used as a model. This image could be a low-resolution image taken from Earth (or an Earth-orbiting telescope). In the second, no model exists or the model is judged to be so divergent from the higher-resolution data as to be unhelpful for reducing data transmission requirements. In this latter case, a down-scaled image is created from the source-scale data and utilized as a model. This low-resolution model must be transmitted along with the MBDT augmentation/correction data.

In both cases, the model image up-scaled to the sourcescale and compared to the source-scale imagery. Deviations between the two are identified and assessed to determine whether they merit transmission inclusion. Several metrics can be included in this assessment. These include the single-pixel difference value as well as the difference values of surrounding pixels. Generally, it is presumed that pixels with a higher level of difference or areas that are more divergent from the model than average represent higher-value data. However, this may not be true in all applications and, thus, the relative weighting of these metrics must be configured for each particular application. Tables 1 and $\mathbf{2}$ show the data transmission requirements for transmitting model updates and the model and model updates, respectively, at various levels of model size and MGQ threshold values.

MGQ is defined as the maximum level of divergence between a data element in the model (e.g., low resolution image) and the high-resolution data. Any variance over the MGQ threshold will result in the inclusion of a corrective message element in the MBDT transmission. However, the MGQ should not be equated to a standard
Table 1. Data transmission required for model updates for different model sizes \& threshold values.

\begin{tabular}{|c|c|c|c|c|c|c|c|}
\hline & & \multicolumn{6}{|c|}{ Model size } \\
\hline & & $5 \%$ & $10 \%$ & $15 \%$ & $20 \%$ & $25 \%$ & $50 \%$ \\
\hline \multirow{7}{*}{ 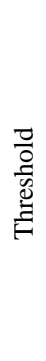 } & $5 \%$ & 79,021 & 68,982 & 61,490 & 61,799 & 61,155 & 32,868 \\
\hline & $7 \%$ & 61,482 & 52,223 & 45,291 & 46,095 & 45,232 & 17,714 \\
\hline & $10 \%$ & 44,545 & 33,598 & 26,961 & 28,350 & 28,192 & 6945 \\
\hline & $15 \%$ & 23,415 & 16,670 & 12,621 & 13,967 & 13,660 & 1815 \\
\hline & $20 \%$ & 13,077 & 7812 & 6095 & 6820 & 7035 & 610 \\
\hline & $30 \%$ & 3500 & 1742 & 1404 & 1838 & 2006 & 263 \\
\hline & $50 \%$ & 337 & 299 & 295 & 311 & 309 & 257 \\
\hline
\end{tabular}

Table 2. Data transmission required for model and model updates for different model sizes \& threshold values.

\begin{tabular}{|c|c|c|c|c|c|c|c|}
\hline & & \multicolumn{6}{|c|}{ Model size } \\
\hline & & $5 \%$ & $10 \%$ & $15 \%$ & $20 \%$ & $25 \%$ & $50 \%$ \\
\hline \multirow{7}{*}{ 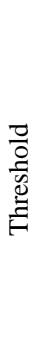 } & $5 \%$ & 79,349 & 70,293 & 64,440 & 67,042 & 69,347 & 65,636 \\
\hline & $7 \%$ & 61,810 & 53,534 & 48,241 & 51,338 & 53,424 & 50,482 \\
\hline & $10 \%$ & 44,873 & 34,909 & 29,911 & 33,593 & 36,384 & 39,713 \\
\hline & $15 \%$ & 23,743 & 17,981 & 15,571 & 19,210 & 21,852 & 34,583 \\
\hline & $20 \%$ & 13,405 & 9123 & 9045 & 12,063 & 15,227 & 33,378 \\
\hline & $30 \%$ & 3828 & 3053 & 4354 & 7081 & 10,198 & 33,031 \\
\hline & $50 \%$ & 665 & 1610 & 3245 & 5554 & 8501 & 33,025 \\
\hline
\end{tabular}

or average level of error. Most data elements (e.g., pixels for image data) will have an error level that is lower than the MGQ threshold.

Tables 1 and $\mathbf{2}$ show that the required level of corrective data declines as the model size is increased (comparative to the size of the source data) and acceptable MGQ is increased. At the highest quality (lowest acceptable MGQ) and smallest model (highlighted on the tables) sizes, the data transmission requirement actually exceeds the requirement to transmit the whole, uncompressed image. The transmission requirements are less for other MGQ threshold and model size combinations.

\subsection{Data Format}

For images, the model change description language consist of three parts: a header, section header, and section data [7]. Data segmentation is utilized to allow for the use of local addressing. Local addressing precludes need ing to transmit several bytes of identifying information for each pixel-change (as would be required in the global context).

\subsubsection{Header}

The header consists of a craft identification field, trans- 
mission identification field, transmission sequence number, date and time stamp field, and a validation field. Each of the above fields is of variable length. The fields are delimited by the ASCII 13 carriage return character. When implemented, the MBDT transmission data will be further encapsulated through lower-level transmission protocols.

The locally unique craft identification field identifies the source craft. This field is included to facilitate (via identifying the original source) a craft relaying data for another. At higher MBTR levels, crafts may also process data. If image data, for example, is processed into a higher-level data product, the processing craft will transmit only results and associated validation data, (based on the data from the lower level craft). The validation data would be stamped with the ID of the collecting craft, while the higher-level data product would be stamped with the ID of the producing craft.

The transmission identification field is required for two reasons. First, it uniquely identifies the transmission. This allows reconstruction of a transmission that spans multiple high level messages. The use of multiple messages is anticipated in cases where high priority data may be transmitted immediately (or with limited delay), and lower-priority is transmitted later (e.g., on a space-available basis).

The transmission identification field also identifies the data type being sent and the object or target to which it relates. The format of this field is not specified, allowing the individual mission designer choose a format best suited to the mission.

The transmission sequence number field identifies subsequent messages that are part of a particular transmission. The messages for individual-pixel changes used for the experiment described herein do not require this. However, for region-level changes, where pixel level changes could be applied over top of them, the order in which to perform the changes is critical.

The validation field stores a hash value or checksum which can be utilized to validate that the data has not been modified (due to interference, error or intentionally) during transmission. It is left to the implementer to determine the validation method best suited for a given mission. This service may be provided by lower level protocols and, if so, this field may be left blank.

\subsubsection{Section Header}

Section headers are utilized for identifying the location of the section inside the image. A section, which is defined by an X, Y coordinate pair, is $256 \times 256$ pixels. Because of this, each local address is represented by a single byte. The section header consists of section location and length fields. The section location field is two fivebyte values. The length field is two bytes.

\subsubsection{Section Data}

Section data follows the section header. The format of section data is data-type specific. For image files, it consists of local coordinates and the change to be made, for each change. Two approaches for section data coordinates can be utilized. If it is expected that few changes will be required (e.g., an average of less than one per line), the coordinate set will consist of one-byte $\mathrm{x}$ and $\mathrm{y}$ coordinates. If it is expected that one or more changes will be required per line, then only the $\mathrm{x}$ coordinate is included. Under this format, lines are delimited by set of all zeros ( $\mathrm{x}$-coordinate $=0$, change value $=$ zeros $)$. As the coordinate system starts from a minimum 1,1 point, the zero value is not ambiguous.

\section{Introduction of Image Compression}

The above (Section 6.1) presumes that the model is a bitmap image. This format is lossless; however, it is not particularly efficient if co-transmission is required. In cases where the image model must be transmitted along with the MBDT data, the utilization of compression may be advantageous. Table 3 shows the transmission requirements for models with different levels of JPEG compression and MGQ threshold values. Table 4 shows the combined transmission requirements for the JPEGcompressed model and the MBDT data for different levels of JPEG compression and MGQ threshold values.

Table 3. MBDT data required for various MGQ threshold levels and levels of JPEG compression.

\begin{tabular}{|c|c|c|c|c|c|c|}
\hline & & \multicolumn{5}{|c|}{ JPEG compression setting } \\
\hline & & 10 & 30 & 50 & 70 & 90 \\
\hline \multirow{5}{*}{ 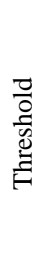 } & $5 \%$ & 36,149 & 18,017 & 12,723 & 7065 & 317 \\
\hline & $10 \%$ & 5917 & 1095 & 483 & 297 & 257 \\
\hline & $15 \%$ & 867 & 275 & 259 & 257 & 257 \\
\hline & $25 \%$ & 263 & 257 & 257 & 257 & 257 \\
\hline & $50 \%$ & 257 & 257 & 257 & 257 & 257 \\
\hline
\end{tabular}

Table 4. MBDT and model data required for various MGQ threshold levels and levels of JPEG compression.

\begin{tabular}{|c|c|c|c|c|c|c|}
\hline & & \multicolumn{5}{|c|}{ JPEG compression setting } \\
\hline & & 10 & 30 & 50 & 70 & 90 \\
\hline \multirow{5}{*}{$\begin{array}{l}\frac{}{0} \\
\frac{0}{0} \\
\frac{0}{d} \\
\frac{1}{E}\end{array}$} & $5 \%$ & 48,003 & 33,847 & 31,368 & 30,094 & 37,356 \\
\hline & $10 \%$ & 17,771 & 16,925 & 19,128 & 23,326 & 37,296 \\
\hline & $15 \%$ & 12,721 & 16,105 & 18,904 & 23,286 & 37,296 \\
\hline & $25 \%$ & 12,117 & 16,087 & 18,902 & 23,286 & 37,296 \\
\hline & $50 \%$ & 12,111 & 16,087 & 18,902 & 23,286 & 37,296 \\
\hline
\end{tabular}


Table 3 shows that at many combinations of levels of JPEG compression and MGQ threshold (highlighted in the table), the JPEG compression alone is suitable to provide the level of quality required. Because of the decision to utilize the data format, for comparison purposes, for section data where a line is included for each line of data (see Section 6.2.3), the minimum possible transmission level required is 257 bytes. However, this equates to no actual corrective data being transmitted. Table 4 shows that the optimal JPEG quality setting varies based on the MGQ threshold desired, with higher levels of MGQ thresholds being most optimally transmitted through the utilization lower JPEG-quality-levels.

\section{Evaluating Comparative Performance}

Its desirable to be able to quantify the quality of images transmitted via a combination of MBDT and data compression. While the MGQ threshold value is a standard that cannot be exceeded on a pixel-by-pixel basis, it does not provide any metric for the error level for the whole image. Such a metric is required for comparing various forms of compression and augmentation. One commonly used metric (e.g., [9-12]) is pixel difference. Table 5 reports the average pixel difference (the sum of the absolute value of all differences in the image divided by the number of pixels present) as a function of threshold value and JPEG compression setting.

With a metric for image quality, it is now possible to evaluate performance relative to file size. Table 6 pre-

Table 5. Average pixel difference values.

\begin{tabular}{|c|c|c|c|c|c|c|}
\hline & & \multicolumn{5}{|c|}{ JPEG compression setting } \\
\hline & & 10 & 30 & 50 & 70 & 90 \\
\hline \multirow{5}{*}{$\begin{array}{l}\text { 등 } \\
\text { 章 } \\
\text { 릍 }\end{array}$} & $5 \%$ & 0.01865 & 0.02026 & 0.01989 & 0.01933 & 0.01254 \\
\hline & $10 \%$ & 0.03473 & 0.02879 & 0.02588 & 0.02250 & 0.01257 \\
\hline & $15 \%$ & 0.03928 & 0.02950 & 0.02607 & 0.02255 & 0.01261 \\
\hline & $25 \%$ & 0.04006 & 0.02952 & 0.02608 & 0.02255 & 0.01261 \\
\hline & $50 \%$ & 0.04007 & 0.02952 & 0.02608 & 0.02255 & 0.01261 \\
\hline
\end{tabular}

Table 6. Image quality as a function of file size.

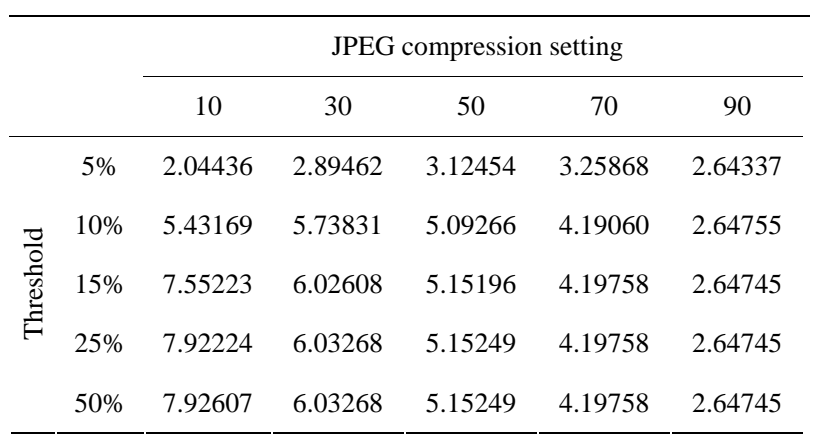

sents this using a metric of the average pixel difference subtracted from one and divided by the file size (in 1/10 megabyte units).

Based on this metric, the low JPEG quality value and high MGQ threshold maximizes the quality/file size metric. This metric, however, fails to consider that a minimum quality level may be required for some applications and that any image with a quality below this level, thus, contains no useful data.

Table 5 shows that the minimum average pixel difference value (APDV) is obtained through the combination of the 5\% MGQ threshold and using a JPEG storage quality level of 90 . This minimum APDV, however, doesn't correlate with the best performance in terms of the image quality as a function of file size (IQFFS) metric. The IQFFS metric is maximized with the lowest JPEG storage quality value $(\mathrm{q}=10)$ and highest MGQ threshold (50\%). The worst performance, is quite logically, the MGQ threshold of 5\% and JPEG storage quality setting of 10 , as at this MGQ level, the most number of MBDT corrections are required to raise the low storage level to the high quality (low MGQ threshold) required.

Table 6 also demonstrates that the IQFFS metric will plateau at points where the JPEG storage quality level meets the MGQ threshold on its own. The JPEG storage quality levels of 30 and 50, for example, plateau (showing no improvement) between the 25\% and 50\% MGQ levels. JPEG quality levels of 70 and 90 show similar effects, starting at the 15\% MGQ threshold. Figures 4-6 depict the visual quality of JPEG quality settings of $\mathrm{q}=$ $10, q=50$ and $q=90$, respectively.

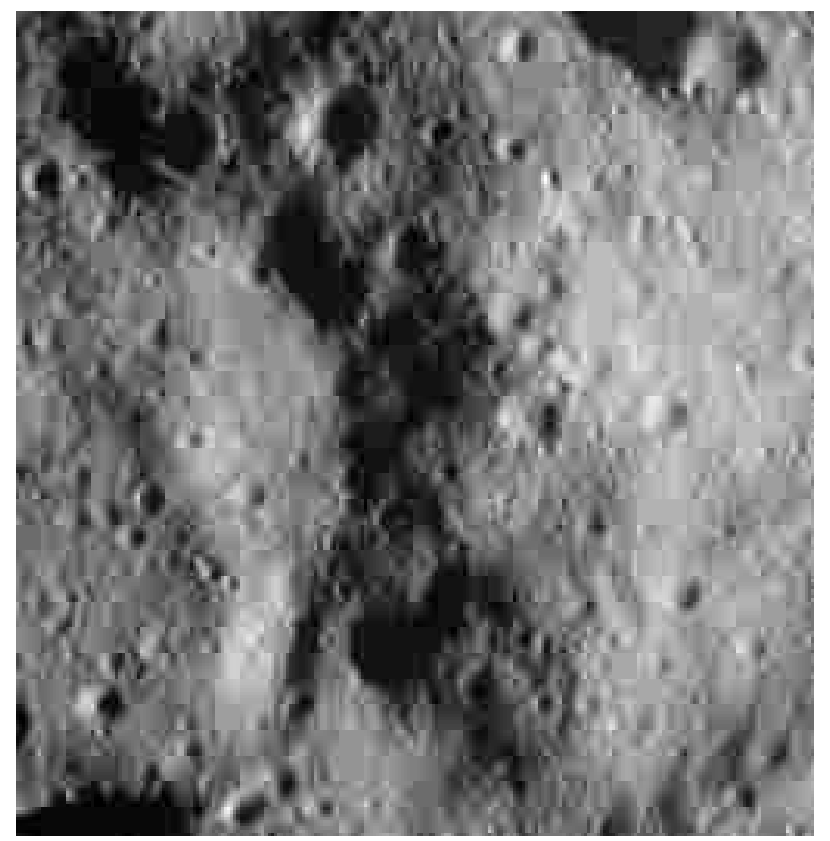

Figure 4. JPEG with image storage quality, $q=10$. 


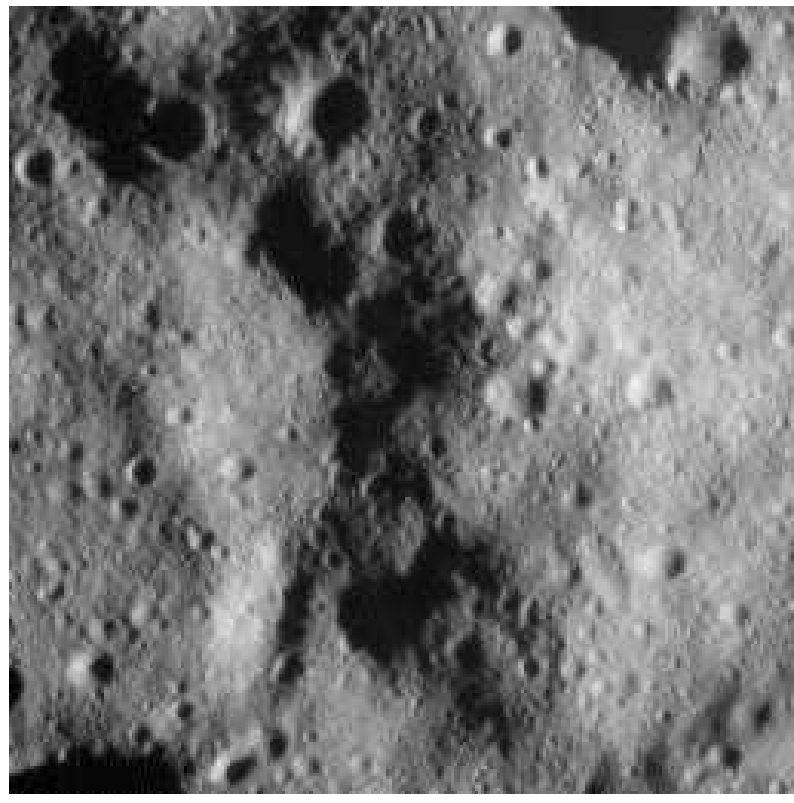

Figure 5. JPEG with image storage quality, $q=50$.

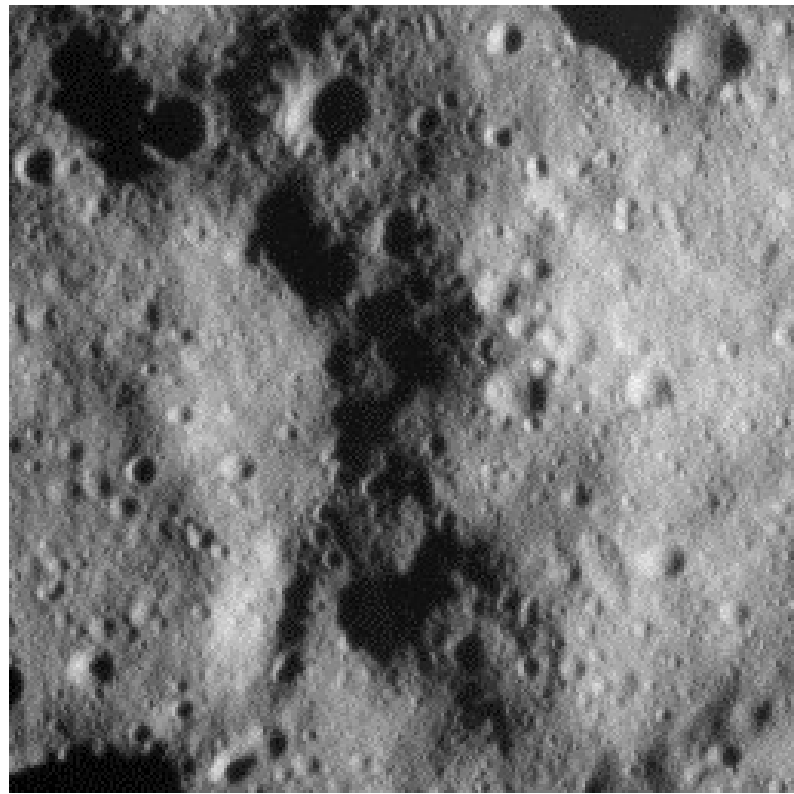

Figure 6. JPEG with image storage quality, $q=90$.

\section{Comparing Compression Techniques}

While a format very similar to the JPEG format has been used in space previously [4], it is only one of numerous compression technologies that are available for use. Table 7 compares the size of several different image formats (including several different levels of JPEG compression). Table 8 presents the average pixel difference values for each image type. Note that several image formats are loss-less or do not result in loss in this instance.

Table 7 shows that the most optimal format to combine with MBDT to achieve the 5\% MGQ standard is JPEG, with a storage quality level of 70 . While the image file size is higher than other JPEG quality levels $(10,50)$, the combination of minimizing file size and MBDT corrections occurs there. The APDV metric, on the other hand, is maximized by the lossless (generally, or in this instance) image formats, as shown in Table 8.

\section{Comparison of Dithering and Non-Dithering Upsizing Methods}

Previous work [7] showed an improvement in performance when dithering occurred due to upsizing an image that could not be upsized on a pixel-to-fixed-multipleof-pixels (where each pixel in the small image corresponded to $\mathrm{a} \times \mathrm{b}$ pixels in the upscaled image). A dithering approach was compared to a non-dithered approach. The data requirement for MBDT transmissions for two image sizes and four threshold levels are presented for both dithered and non-dithered upsizing in Table 9. In all cases, the non-dithered approach out-performed the dithered approach. Given the prior performance [7], additional investigation will be conducted to evaluate the comparative performance of various dithering techniques as a way of possibly further enhancing MBDT.

\section{Combining Image Compression and Reduced Resolution}

Combining image compression and a reduced model image size maximizes the IQFFS metric. Table 10 shows the data levels for MBDT messages required to reach a

Table 7. File sizes for different image formats and associated MBDT data.

\begin{tabular}{|c|c|c|c|c|c|c|c|}
\hline & BMP & PNG & TIFF & JPG-10 & JPG-50 & JPG-70 & JPG-90 \\
\hline Base & 198,458 & 38,958 & 44,290 & 11,854 & 18,645 & 23,029 & 37,039 \\
\hline $5 \%$ MGQ & 198,458 & 38,958 & 44,290 & 48,003 & 31,368 & 30,094 & 37,356 \\
\hline
\end{tabular}

Table 8. Average pixel difference for different image formats and with the use of MBDT augmentation.

\begin{tabular}{ccccccccc}
\hline & BMP & PNG & TIFF & JPG-10 & JPG-50 & JPG-70 & JPG-90 \\
\hline Base & 0 & 0 & 0 & 0.03982 & 0.02563 & 0.02198 & 0.01159 \\
5\% MGQ & 0 & 0 & 0 & 0.01865 & 0.01989 & 0.01933 & 0.01254 \\
\hline
\end{tabular}


Table 9. Comparison of dithering-based and non-dithering upsizing approaches.

\begin{tabular}{|c|c|c|c|c|c|}
\hline & & \multicolumn{2}{|c|}{ Dithered } & \multicolumn{2}{|c|}{ Not Dithered } \\
\hline & & $50 \%$ & $25 \%$ & $50 \%$ & $25 \%$ \\
\hline \multirow{4}{*}{ 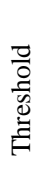 } & $5 \%$ & 47,365 & 70,225 & 37,971 & 61,101 \\
\hline & $10 \%$ & 15,873 & 35,161 & 10,865 & 28,165 \\
\hline & $15 \%$ & 5739 & 17,653 & 3793 & 13,647 \\
\hline & $20 \%$ & 2255 & 8599 & 1413 & 7031 \\
\hline
\end{tabular}

Table 10. MBDT message size required to reach various MGQ threshold level for multiple image sizes and levels of JPEG quality.

\begin{tabular}{|c|c|c|c|c|c|}
\hline & & \multicolumn{2}{|c|}{ JPEG q $=70$} & \multicolumn{2}{|c|}{ JPEG q $=90$} \\
\hline & & $50 \%$ & $25 \%$ & $50 \%$ & $25 \%$ \\
\hline \multirow{5}{*}{ 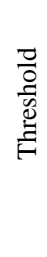 } & $5 \%$ & 41,440 & 57,078 & 34,882 & 51,540 \\
\hline & $10 \%$ & 9292 & 21,312 & 6882 & 17,788 \\
\hline & $15 \%$ & 1862 & 7876 & 1430 & 6618 \\
\hline & $20 \%$ & 492 & 2892 & 380 & 2620 \\
\hline & $25 \%$ & 290 & 1170 & 262 & 1126 \\
\hline
\end{tabular}

given MGQ threshold. Table 11 combines the MBDT messages and model image file size. Table $\mathbf{1 2}$ presents the average pixel difference for each JPEG quality level, threshold MGQ level, and model image size. Table 13 presents the resulting image quality as a function of file size, using the IQFFS metric previously described. The combination of JPEG 70 storage quality, a $25 \% \times 25 \%$ height and width and a MGQ threshold of $25 \%$ maximizes the performance, among the tested scenarios, producing an IQFFS metric value of 9.06. Note that this combined approach out-performs the JPEG-compressiononly approach: producing a superior IQFFS value at a MGQ threshold of $25 \%$ to what was produced by JPEG compression at MGQ of 50\%.

The best performing combination, in terms of IQFFS, is neither the best performing in terms of MBDT message size or APDV. MBDT message size, the metric that one would seek to minimize if the model was not being co-transmitted, is minimized by the JPEG q $=90$, model size of $50 \% \times 50 \%$ height and width at all MGQ threshold levels. The best performance for APDV is provided by the JPEG q $=70$, model size of $25 \% \times 25 \%$ height and width at the MGQ 5\% threshold level. Note that at higher MGQ levels, the best performing APDV for the level is provided by the JPEG q $=90$, model size $50 \% \times$ $50 \%$ height and width. It appears that given the significant number of MBDT updates required to meet the 5\% MGQ threshold, the condition that required the most modifications (JPEG q $=70$, model size of $25 \% \times 25 \%$ height and width) out performed due to a near brute-force
Table 11. MBDT messages and image size required to reach various MGQ threshold level for multiple image sizes and levels of JPEG quality.

\begin{tabular}{|c|c|c|c|c|c|}
\hline & & \multicolumn{2}{|c|}{$\mathrm{JPEG} \mathrm{q}=70$} & \multicolumn{2}{|c|}{ JPEG q $=90$} \\
\hline & & $50 \%$ & $25 \%$ & $50 \%$ & $25 \%$ \\
\hline \multirow{5}{*}{ 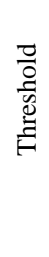 } & $5 \%$ & 54,305 & 66,300 & 51,422 & 61,556 \\
\hline & $10 \%$ & 22,157 & 30,534 & 23,422 & 27,804 \\
\hline & $15 \%$ & 14,727 & 17,098 & 17,970 & 16,634 \\
\hline & $20 \%$ & 13,357 & 12,114 & 16,920 & 12,636 \\
\hline & $25 \%$ & 13,155 & 10,392 & 16,802 & 11,142 \\
\hline
\end{tabular}

Table 12. Average pixel difference at various MGQ threshold level for multiple image sizes and levels of JPEG quality.

\begin{tabular}{|c|c|c|c|c|c|}
\hline & & \multicolumn{2}{|c|}{$\mathrm{JPEG} \mathrm{q}=70$} & \multicolumn{2}{|c|}{ JPEG q $=90$} \\
\hline & & $50 \%$ & $25 \%$ & $50 \%$ & $25 \%$ \\
\hline \multirow{5}{*}{ 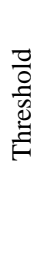 } & $5 \%$ & 0.01810 & 0.01624 & 0.01898 & 0.01679 \\
\hline & $10 \%$ & 0.03560 & 0.03604 & 0.03411 & 0.03546 \\
\hline & $15 \%$ & 0.04244 & 0.04862 & 0.03913 & 0.04590 \\
\hline & $20 \%$ & 0.04422 & 0.05517 & 0.04048 & 0.05117 \\
\hline & $25 \%$ & 0.04455 & 0.05810 & 0.04068 & 0.05370 \\
\hline
\end{tabular}

Table 13. Image quality as a function of file size for various MGQ threshold level for multiple image sizes and levels of JPEG quality.

\begin{tabular}{|c|c|c|c|c|c|}
\hline & & \multicolumn{2}{|c|}{ JPEG q $=70$} & \multicolumn{2}{|c|}{$\mathrm{JPEG} \mathrm{q}=90$} \\
\hline & & $50 \%$ & $25 \%$ & $50 \%$ & $25 \%$ \\
\hline \multirow{5}{*}{ 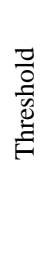 } & $5 \%$ & 1.80812 & 1.48381 & 1.90777 & 1.59725 \\
\hline & $10 \%$ & 4.35256 & 3.15699 & 4.12385 & 3.46905 \\
\hline & $15 \%$ & 6.50206 & 5.56425 & 5.34705 & 5.73583 \\
\hline & $20 \%$ & 7.15568 & 7.79947 & 5.67090 & 7.50894 \\
\hline & $25 \%$ & 7.26301 & 9.06369 & 5.70956 & 8.49312 \\
\hline
\end{tabular}

(replacing more pixels with actual values) approach. The best performing in terms of the IQFFS metric does correlate with the best performing condition in terms of file size: JPEG q $=70,25 \% \times 25 \%$ height and width with a 25\% MGQ threshold in both cases.

\section{Evaluation of Combined Model}

The MBDT approach outperforms JPEG compression in terms of the IQFFS metric. Based on the trends observed, it would seem likely that even higher IQFFS scores could be generated by testing lower JPEG storage quality levels and smaller model sizes.

The IQFFS metric, while being well-correlated with desirable performance trends effectively incorporates an 
arbitrary weighting between the average pixel difference and the file size. The metric will increase with decreased file size or decreased pixel error (APDV); however, the comparative level of change for a change in file size versus error can be set at numerous levels. The comparative benefit of quality versus transmission cost will clearly need to be evaluated by application/mission planners. For this reason, the components of the IQFFS metric have been presented for all of the various experiments performed, allowing the reader to review the performance of the various experimental conditions in terms of his or her own preferred weighting between size and quality.

Figures 7 and 8 show the best performing images in terms of the IQFFS and APDV metrics, respectively. Figure 9 shows the best performing image in terms of MBDT message minimization. A visual comparison of these images clearly indicates that IQFFS is not an appropriate metric for maximizing human viewing pleasure. However, the images presented in both Figures 8 and 9 would appear suitable. Figure 9, in particular, shows the utility of using MBDT to provide higher resolution imagery based on the enhancement of a pre-shared model.

\section{Conclusions and Future Work}

The work presented demonstrates the value of using model based data transmission. In the case where a pre-shared, lower-resolution model is available, data transmission can be reduced significantly. Even if a model is not available, however, MBDT can be utilized to maximize the utility of the available bandwidth. Via

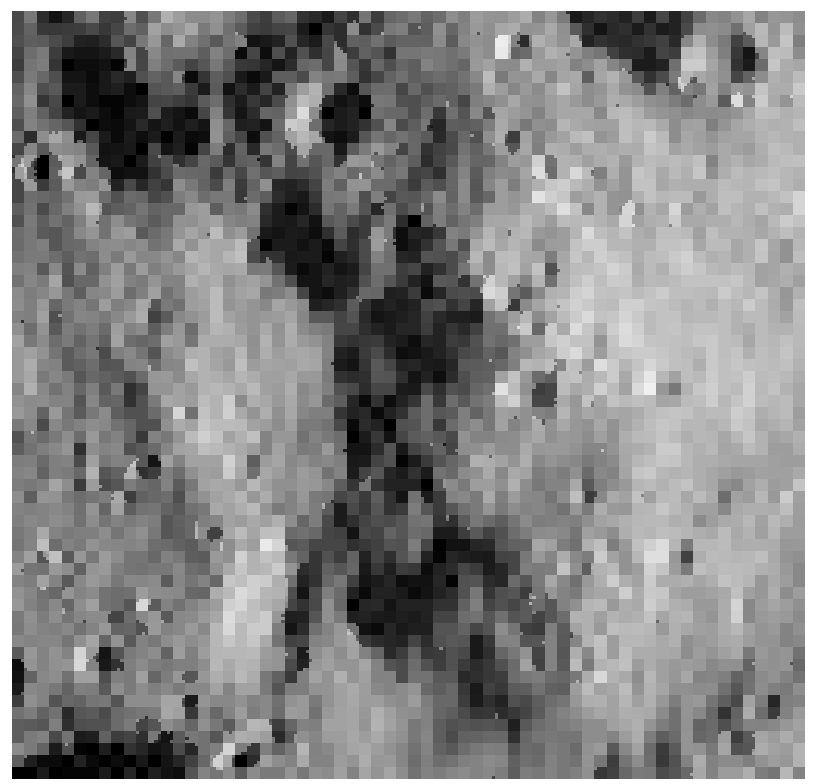

Figure 7. Best performing image in terms of IQFFS, JPEG $\mathbf{q}=70$, MGQ threshold $=25 \%$ and model size of $25 \% \times$ $25 \%$ height and width.

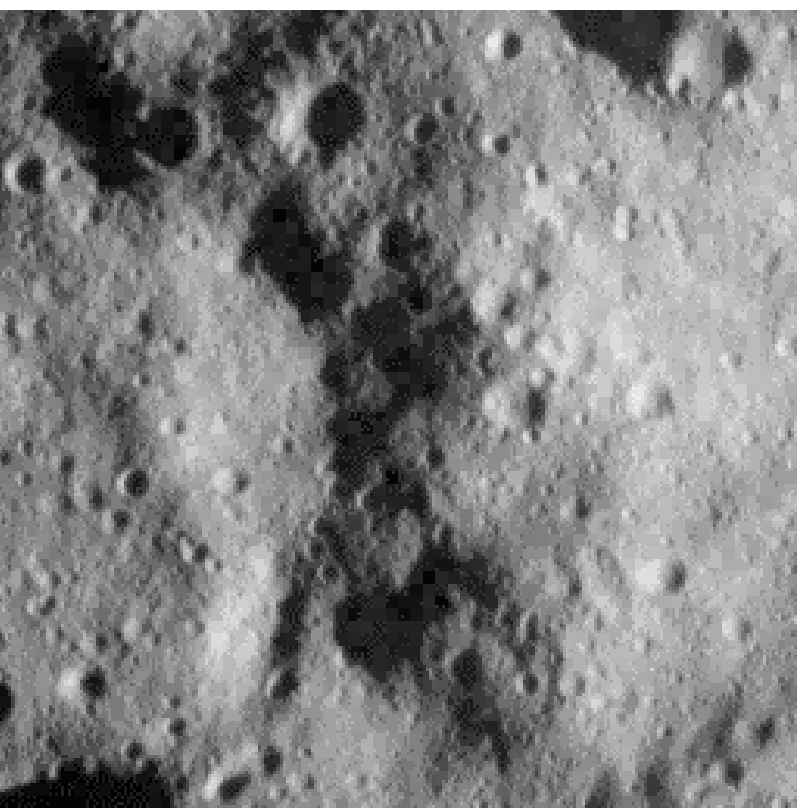

Figure 8. Best performing image in terms of MGQ, JPEG q = 70, MGQ threshold $=5 \%$ and model size of $25 \% \times 25 \%$ height and width.

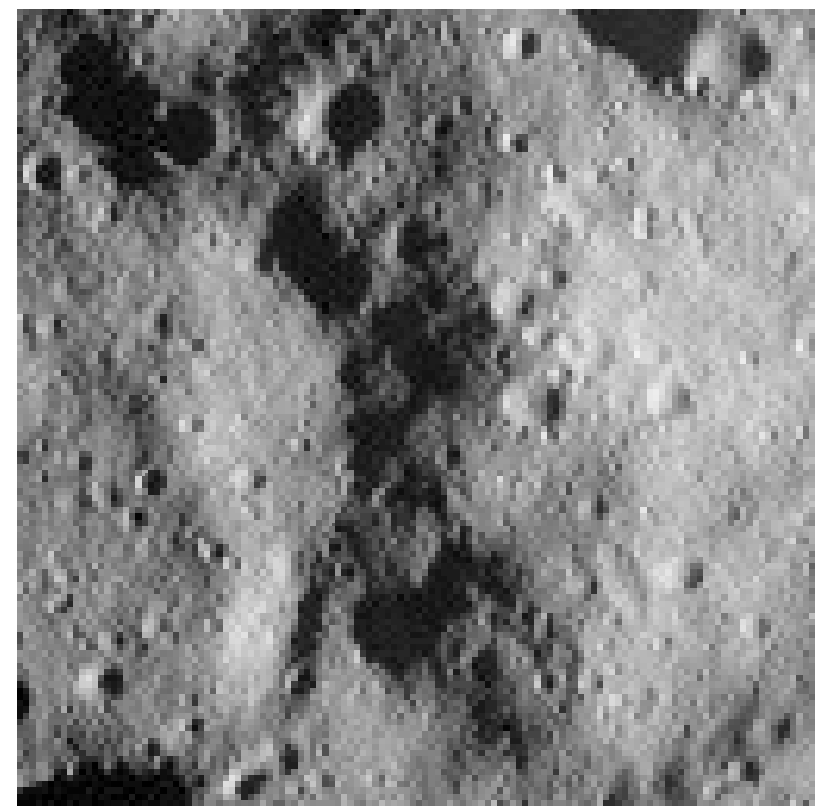

Figure 9. Best performing image in terms of MBDT message minimization, JPEG q $=90$, MGQ threshold $=25 \%$ and model size of $50 \% \times 50 \%$ height and width.

utilizing MBDT, a minimum level of quality can be guaranteed and/or features can be prioritized based on their importance (which is presumed to be a function of a given area's discrepancy from the model).

While the work described herein deals with reducing data transmission for an unknown, unchanging environment, MBDT also has applications in a known, changing environment. Consider a disaster response scenario, for 
example. High-resolution data very likely exists for the area (possibly even taken by the same satellite that will re-image it after the disaster). Using MBDT, differences can be identified and prioritized based on the level of difference. Not only does this reduce the bandwidth required for transmission (allowing responders to obtain the data more quickly), it also highlights areas where focus is needed (e.g., those areas that have presumably been impacted by the disaster).

Future work will focus on evaluating MBDT with a larger data set, evaluating its use in a disaster scenario and characterizing the performance (in terms of knowledge gained per byte transmitted) of other MBTR levels. This will allow the relative performance the MBTR levels to be compared in terms of a common metric. Further, application-specific performance metrics will be explored to facilitate an analysis of MBDT/MBTR performance in terms of user application performance metrics.

\section{Acknowledgements}

Small satellite development work at the University of North Dakota is funded by the North Dakota Space Grant Consortium, the UND Faculty Research Seed Money Committee and North Dakota NASA EPSCoR.

\section{REFERENCES}

[1] M. Bozzi, M. Cametti, M. Fornaroli, P. Maguire, S. Marti, M. Pasian, K. Perregrini and S. Rawson, "Future Architectures for European Space Agency Deep-Space Ground Stations [Antenna Applications Corner]," Antennas and Propagation Magazine, Vol. 54, No. 1, 2012, pp. 254-263. doi:10.1109/MAP.2012.6202560

[2] J. Schou, et al., "Design and Ground Calibration of the Helioseismic and Magnetic Imager (HMI) Instrument on the Solar Dynamics Observatory (SDO)," Solar Physics, Vol. 275, No. 1-2, 2012, pp. 229-259.

doi:10.1007/s11207-011-9842-2
[3] D. Barret, et al., "The High Time Resolution Spectrometer (HTRS) aboard the International X-Ray Observatory (IXO)," Proceedings of SPIE, Vol. 2011, No. 3, p. $77321 \mathrm{M}$.

[4] K. Cheung, M. Belongie and K. Tong, "End-to-End System Consideration of the Galileo Image Compression System,” TDA Progress Report 42-126, 1996.

[5] R. Lutz, "Software Engineering for Space Exploration," IEEE Computer, Vol. 44, No. 10, 2011, pp. 41-46. doi:10.1109/MC.2011.264

[6] L. Faria, L. Fonseca and M. Costa, "Performance Evaluation of Data Compression Systems Applied to Satellite Imagery,” Journal of Electrical and Computer Engineering, Vol. 2012, No. 2012, 2012, 15 p. doi:10.1155/2012/471857

[7] J. Straub, "Reducing Link Budget Requirements with Model-Based Transmission Reduction Techniques," Proceedings of the 26th Annual AIAA/USU Conference on Small Satellites, Logan, 13-16 August 2012.

[8] J. Straub, "Increasing Interplanetary CubeSat Mission Science Return with Model Based Transmission Reduction,” The 1st Interplanetary CubeSat Workshop, Boston, 2012.

[9] M. Trifas and J. Straub, “A Comparison of Techniques for Super-Resolution Evaluation," Proceedings of the IS\&T/SPIE Electronic Imaging Conference, Burlingame, 22 January 2012. doi:10.1117/12.912172

[10] M. Trifas and J. Straub, "Super Resolution: A Database Driven Inference Approach," Proceedings of the 15th World Multi-Conference on Systemics, Cybernetics and Informatics, Orlando, 19-22 July 2011.

[11] M. Kharrazi, H. Sencar and N. Memon, "Blind Source Camera Identification,” Proceedings of the 2005 International Conference on Image Processing, Genoa, 11-14 September 2005, pp. 69-72.

[12] Z. Whang, A. Bovik, H. Sheikh and E. Simoncelli, "Image Quality Assessment: From Error Visibility to Structural Similarity," IEEE Transactions on Image Processing, Vol. 13, No. 4, 2004, pp. 600-612. 